\title{
"Roll over Beethoven ». La sociologie des arts aux prises avec le polythéisme des valeurs
}

André Ducret

\section{(2) OpenEdition}

Journals

Édition électronique

URL : http://journals.openedition.org/ress/540

DOI : $10.4000 /$ ress.540

ISSN : $1663-4446$

Éditeur

Librairie Droz

Édition imprimée

Date de publication : 1 septembre 2003

Pagination : 103-116

ISBN : 2-600-00878-0

ISSN : 0048-8046

Référence électronique

André Ducret, « «Roll over Beethoven ». La sociologie des arts aux prises avec le polythéisme des valeurs », Revue européenne des sciences sociales [En ligne], XLI-126 | 2003, mis en ligne le 30 novembre 2009, consulté le 02 mai 2019. URL : http://journals.openedition.org/ress/540 ; DOI $10.4000 /$ ress. 540 


\section{André DUCRET}

\section{«ROLL OVER BEETHOVEN.» LA SOCIOLOGIE DES ARTS AUX PRISES AVEC LE POLYTHÉISME DES VALEURS}

Dans un texte où il s'interroge sur l'objectivité des valeurs artistiques, Raymond Boudon pose la question de savoir comment il se fait qu'aujourd'hui encore, on continue de valoriser des œuvres apparues dans des cultures révolues (Boudon, 1994). C'était là, on s'en souvient, le problème que rencontrait déjà Karl Marx face à l'art de la Grèce antique, problème qu'il croyait résolu une fois le respect que nous inspire l'art du passé assimilé à l'attrait éternel qu'éprouve pour son enfance l'homme mûr qui sait que ces temps heureux jamais ne reviendront (Marx \& Engels, 1954) ${ }^{1}$. En revanche, pour Boudon, si nous estimons aujourd'hui encore que l'art de Rembrandt ou celui de Rodin présentent des qualités telles qu'elles justifient l'admiration que nous leur portons, c'est que notre jugement se fonde sur ce qu'il nomme des «raisons objectives». Du même coup, il n'hésite pas à faire de Georg Simmel un éminent sociologue de l'art pour avoir compris et formulé ce qui faisait la grandeur de ces deux artistes de sorte qu'avec lui, écrit-il, «l'esthétique s'intègre facilement avec la sociologie de l'art telle qu'il la conçoit» (Boudon, 1994: 252, n. 1). Or, non seulement ladite intégration soulève à nos yeux plus de problèmes qu'elle n'en résout, mais on peut se demander si le fait de recourir à des catégories comme celles de «raisons objectives» ou de «raisons fortes » ne risque pas de ramener à un raisonnement du type: ce que je crois beau l'est effectivement, raison pour laquelle je le crois beau.

Certes, il n'est jamais inutile de se rappeler que tout jugement de valeur, avant d'être causé, est d'abord fondé si bien qu'en s'interdisant de s'interroger sur ce qui le fonde, le sociologue risque de passer à côté de son objet. Mais les raisons

Dans son Introduction à la critique de l'économie politique, Marx (1954: 184-185) avance que « la difficulté ne consiste pas à comprendre que l'art grec et l'épopée soient liés à certaines formes du développement social. La difficulté consiste à comprendre qu'ils puissent nous procurer encore des jouissances esthétiques et soient considérés à certains égards comme norme et comme modèles inimitables.» Et de poursuivre: «Un homme ne peut redevenir enfant sans tomber en enfance. Mais ne se réjouit-il pas de la naïveté de l'enfant, et ne doit-il pas lui-même aspirer à reproduire, à un niveau supérieur, sa vérité? Est-ce que, dans la nature enfantine, le caractère propre de chaque époque ne revit pas dans sa vérité naturelle? Pourquoi l'enfance sociale de l'humanité, au plus beau de son épanouissement, n'exercerait-elle pas, comme une phase qui ne reviendra jamais plus, un éternel attrait? Il y a des enfants mal élevés et des enfants précoces. Beaucoup de peuples anciens appartiennent à cette catégorie. Les Grecs étaient des enfants normaux. L'attrait qu'a pour nous leur art n'est pas en contradiction avec le faible développement de la société où il a grandi. Il en est plutôt le résultat; il est plutôt lié indissolublement au fait que les conditions sociales inachevées où cet art est né, et où il pouvait seulement naître, ne pourront jamais plus revenir.». 
formulées à l'appui de n'importe quelle appréciation suffisent-elles à l'expliquer? Car d'où vient que l'un croie fermement à la valeur de telle œuvre ou de tel artiste alors que l'autre refuse de leur accorder la moindre considération? Et comment s'acquitter de l'expérience que beaucoup d'entre nous avons faite à savoir que nos goûts et nos jugements évoluent selon les situations que nous traversons si bien que les arguments que nous tenions naguère pour excellents se trouvent aujourd'hui relégués au rang d'erreurs de jeunesse?

Soucieux à juste titre de combattre un relativisme toujours prompt à surgir de sa boîte dès qu'il est question de valeurs, Raymond Boudon s'en prend avec vigueur à ce qu'il stigmatise comme un conventionnalisme dans l'incapacité d'expliquer «qu'il existe des œuvres et des artistes considérés comme 'classiques', des hiérarchies relativement stables des œuvres et des artistes, ou encore qu'il est relativement facile d'exporter des œuvres d'art d'une culture à une autre » (Boudon, 1994: 260-261). D'après lui, un Howard S. Becker et ses «mondes de l'art» ne permettent de rendre compte ni de la façon dont des valeurs s'établissent ni des raisons pour lesquelles elles se maintiennent si bien qu'à cultiver le paradigme constructiviste, la sociologie des arts s'engagerait dans une impasse alors même que des auteurs comme Georg Simmel ou Pierre Francastel lui ouvrirent jadis des voies plus prometteuses. Et Boudon de conclure sur des considérations censées justifier de manière objective, explicite et définitive ses préférences pour Klee plutôt que pour Mondrian, ou encore l'émotion qu'il ressent et que, une fois d'accord avec lui, nous pourrions également ressentir à l'écoute de Chostakovitch comme s'il était possible, voire souhaitable de séparer le jugement de valeur de celui de goût. Mais à vouloir échapper aux pièges de ce qu'il condamne comme une démarche réductionniste, ne retombe-t-il pas alors dans un propos plus proche de l'esthétique que de la sociologie?

\section{DU RELATIVISME}

Une longue fréquentation des auteurs que Raymond Boudon érige en fers de lance d'une offensive en règle contre une démarche qui se refuserait à concéder la moindre place aux qualités propres à l'œuvre d'art dans l'explication des raisons pour lesquelles nous l'admirons me rend particulièrement sensible à une argumentation à laquelle je ne puis toutefois souscrire. Le projet scientifique qui caractérise notre discipline ne consiste-t-il pas, sauf à se renier sur l'essentiel, à marquer les différences entre groupes, contextes et moments plutôt que de parier sur leur réduction au cas où certaines conditions seraient réunies? Se défier de tout discours à prétention universaliste me semble la racine même du raisonnement sociologique sans qu'un tel principe conduise nécessairement au relativisme, ce que confirme à sa façon Jacques Coenen-Huther lorsqu'il souligne que «comme artifice méthodologique, le postulat de rationalité n'a prouvé son efficacité qu'assorti d'une conscience aiguë de la diversité des rationalités » (Coenen-Huther, 2001: 237). La vocation de la sociologie serait donc, sinon relativiste, du moins relationnelle dès lors qu'elle s'attache à mettre en lumière les liens supposés entre divers ordres de phénomènes, l'action réciproque des uns sur les autres, ou encore les interdépendances au fondement du social. 
Le fait de déclarer «transsubjectives » plutôt qu'objectives les raisons au nom desquelles tomber d'accord sur la grandeur de Mozart ou sur celle de Molière permet-il de lever tout malentendu? Et la frontière entre esthétique et sociologie devient-elle plus nette pour autant?

De prime abord, répétons-le, la décision de ne pas négliger, dans l'analyse des représentations ou des croyances, l'objet même sur lequel portent ces dernières, paraît légitime et l'on conçoit mal que la sociologie s'interdise par principe tout propos sur l'œuvre d'art. Ainsi Pierre Demeulenaere plaide-t-il à son tour pour qu'on «accorde un sort à la pertinence des représentations esthétiques dans une relation directe à leur objet plutôt que de réduire la possibilité de cette pertinence à des causalités de rang supérieur ou à des facteurs extérieurs à l'esthétique proprement dite» même si, s'empresse-t-il d'ajouter, «ceux-ci, bien entendu, existent» (Demeulenaere, 1998: 292). Examiner comment, à partir de la spécificité de leur objet, se constituent des jugements de valeur, des représentations ou des croyances, telle serait la tâche du sociologue qui, confronté à une pluralité d'opinions et d'attitudes radicalement opposées parfois, s'efforcerait de comprendre comment un accord peut néanmoins se faire et résister à l'usure du temps. Ce même sociologue aurait à s'interroger, en particulier, sur la part prise par le discours savant dans ce processus de cristallisation de la valeur. Or, lorsqu'il cherche à dégager les critères au nom desquels Ernst H. Gombrich décide de l'importance de tel ou tel tableau pour l'évolution de la peinture occidentale, Demeulenaere découvre que, s'il est concevable d'en dresser la liste, ces critères varient selon les œuvres auxquelles ils s'appliquent: tantôt l'historien de l'art met en avant la maîtrise technique dont fait preuve l'artiste, tantôt il privilégie l'originalité de l'œuvre, tantôt encore il exalte sa fidélité au réel. Mais d'où vient que, chez ce seul et même auteur, et sans jamais qu'il succombe à l'incohérence ou à l'arbitraire, telle raison soit invoquée ici et telle autre là?

Faut-il en conclure que l'œuvre d'art impose d'elle-même qu'on la juge selon tel ou tel critère? Et si tel était le cas, que penser des nombreuses controverses entre historiens de l'art - pour ne pas parler de celles, quelquefois féroces, impliquant d'autres publics? Plutôt que de supposer qu'à la condition - toute virtuelle - d'être dans la position qu'occupait Gombrich, nous verrions l'histoire de l'art du même œil que lui, ne vaut-il pas mieux s'intéresser à la diversité des situations dans lesquelles, de fait, se construisent les jugements de valeur, aux polémiques, aux modes et autres redécouvertes qui constituent un terrain d'observation privilégié pour le sociologue? Ce que Jacques Coenen-Huther ressent comme «une certaine circularité entre la transsubjectivité et l'adhésion collective» (CoenenHuther, 1999: 100)² porte ainsi à conclure à l'impossibilité de ne pas trancher entre deux perspectives: l'une, esthétique, vouée à décliner les qualités de l'objet, et l'autre, sociologique, centrée sur les rapports des sujets audit objet, dont on postulera qu'ils procèdent des relations que ces sujets entretiennent les uns avec les autres. De ce point de vue, les textes que Georg Simmel consacre à Rembrandt

Une «circularité» que Jacques Coenen-Huther (1999: 100) décrit comme suit: «Une croyance 'fait sens' parce qu'elle s'appuie sur des raisons fortes. Les raisons sont perçues comme 'fortes' parce qu'elles sont largement partagées. Elles sont partagées par le grand nombre - et deviennent dès lors un facteur de causalité - parce qu'elles sont ressenties comme fortes ». 
ou à Rodin, s'ils recoupent ses préoccupations épistémologiques ou sa définition de la sociologie, n'en relèvent pas moins de l'esthétique ou - genre alors en vogue - de la philosophie de la culture plutôt que de la sociologie des arts, notamment si l'on songe aux diverses recherches empiriques qui se sont développées depuis dans ce domaine complexe et foisonnant qu'est celui de la réception.

Ailleurs, Raymond Boudon n'hésite pas à faire d'Howard S. Becker le «Kuhn de la sociologie de l'art», car écrit-il, «tout comme les sociologues des sciences, Becker présente, lui aussi, l'anthropologie comme la reine des sciences: les mondes de l'art doivent s'analyser comme des tribus et leurs croyances comme celles des Azandé» (Boudon, 1995: 485). Là encore, la charge est rude dans la mesure où l'usage que fait ce dernier de la notion - certes polysémique - de «conventions» demeure limité et vise pour l'essentiel à démontrer que toute œuvre d'art est le produit d'une activité collective qui repose sur une compréhension mutuelle et sur le recours à un certain nombre de règles de comportement le plus souvent implicites. Ainsi que le remarque Alban Bouvier, il ne s'agit pas de «faire plus, à travers cela, qu'une sociologie des professions artistiques et des réseaux sociaux qui permettent à l'œuvre d'art de se réaliser et de s'accomplir, les discours de légitimation y aidant» (Bouvier, 1998: 323) sans qu'on puisse étiqueter le sociologue américain comme un thuriféraire du relativisme postmoderne.

Pour Becker, les mondes de l'art influent sur la production des œuvres, sur leur diffusion et, également, sur leur réception en leur attribuant certaines qualités ou en leur prêtant telle ou telle signification. Il s'ensuit que, faute d'un accord sur les critères au nom desquels juger de la valeur de ces œuvres, la possibilité même d'une activité en commun et l'émergence d'un réseau d'acteurs voués à leur promotion s'avèrent compromises. Cet accord, ces conventions ou convictions partagées peuvent aussi bien naître d'un débat ouvert entre les acteurs que s'imposer à eux parce que la tradition veut qu'on considère les choses ainsi ou parce qu'il ne vient à l'esprit de personne de les aborder sous un autre angle. Voilà pourquoi, selon Becker, il convient d'examiner de près les interactions qui cimentent le monde de l'art afin de comprendre comment se construit la réputation de l'artiste ou la reconnaissance de l'œuvre. Certes, le fait de différencier le court terme du long terme peut s'avérer utile afin d'expliquer comment des effets de mode peuvent s'attacher provisoirement à tel artiste ou à telle œuvre ${ }^{3}$, mais rares sont par contre les situations où l'un ou l'autre serait parvenu à se faire reconnaître de manière durable sans l'activité de mondes de l'art qui soient en mesure ne seraitce que d'en entretenir le culte ou la mémoire (Lang, 1990).

Parce qu'elles prennent à contre-pied le postulat à l'origine d'une part essentielle de la sociologie des arts, les objections que formule Raymond Boudon invitent à démontrer que le scepticisme critique ne conduit pas nécessairement au relativisme normatif - démonstration à laquelle cet auteur fournit d'ailleurs luimême plusieurs arguments de poids, en particulier lorsqu'il oppose à l'idée selon

Selon Boudon (1994: 259), «il arrive bien sûr que les réseaux constituant les 'mondes de l'art' puissent un temps parvenir à valoriser ou à dévaloriser un auteur qui ne le mérite pas. Mais on n'a jamais vu une œuvre s'imposer durablement sous l'action des seuls 'mondes de l'art'. Malgré les subventions et les efforts déployés en leur faveur, bien des produits de la musique d'avant-garde passent justement pour peu de valeur». 
laquelle aucun point de vue ne saurait jamais prévaloir parmi l'infinité des interprétations possibles d'un même événement, la quête obstinée de la vérité scientifique, fondée sur la critique réciproque et sur la mise à l'épreuve systématique des théories en présence (Boudon, 1996).

Le problème que pose à la sociologie l'existence d'œuvres dites «classiques » ou le fait qu'un accord puisse se faire sur la grandeur d'un artiste renvoie à ce que Boudon décrit - à l'aide d'une métaphore vinicole - comme un processus de décantation au cours duquel des idées ou des valeurs s'imposent peu à peu parce que perçues comme s'appuyant sur des raisons fortes. Dans cette perspective, il s'agit d'analyser comment, au fil du temps, se constituent des ordres, des classements et des catégories qui, à partir d'un certain moment, vaudraient pour ainsi dire une fois pour toutes si bien que finirait par être qualifiée de classique l'œuvre consacrée au nom de raisons assez solides pour n'être plus mises en question si ce n'est par quelques esprits égarés, la tâche du sociologue - comme, du reste, de l'historien - étant alors de mettre en lumière les conditions dans lesquelles se serait forgé ce consensus définitif ou quasi-objectif.

A cette définition des classiques, on peut cependant préférer celle avancée par George Steiner, d'autant plus pertinente qu'à l'inverse de la précédente, elle se fonde sur l'impossibilité d'épuiser le sens des grandes œuvres. Est un classique, selon Steiner, une «forme signifiante qui nous 'lit'». Il nous lit plus que nous le lisons (l'écoutons, le percevons)» car, précise-t-il, « chaque fois que nous sommes aux prises avec lui, le classique nous questionnera. Il mettra au défi nos ressources de conscience et d'intelligence, d'esprit et de corps (si grande est la part corporelle de l'esthétique première et même de la réponse intellectuelle). Le classique nous demandera: 'As-tu compris?; as-tu ré-imaginé de manière responsable?; es-tu prêt à agir sur les questions, sur les potentialités d'être transformé, enrichi, que j'ai posées'» (Steiner, 1998: 32-33). Une définition qui, à l'évidence, ne doit rien à la sociologie, mais qui pousse à examiner les diverses interprétations données de l'œuvre selon les contextes, les lieux ou les moments tout en laissant ouverte - il faut le souligner - la possibilité que naissent des réponses mieux fondées que d'autres.

Pour Steiner, les œuvres destinées à demeurer des classiques, celles qui traversent le temps, sont celles qui ne se laissent jamais interpréter de manière définitive. Par contre, «en musique, en littérature ou dans les arts, les vétilles, le travail éphémère se trahissent par le fait qu'on puisse les ranger en catégories et souscatégories une fois pour toutes. En un sens parfaitement rationnel et pragmatique, un acte sérieux de signification - verbal, imagé ou tonal - ne saurait être épuisé par quelque sommation interprétative que ce soit. On ne saurait l'anatomiser ni le figer à une place donnée. Il n'est de lecture, au sens le plus large du terme, il n'est de cartographie herméneutico-critique, qui ne demeure provisoire, incomplète et possiblement erronée» (Steiner, 1998: 35).

De ce point de vue, le «classique» demeure une œuvre ouverte et sa compréhension, à jamais inachevée, sans cesse recommencée sur la base ou à rebours des interprétations précédentes de sorte que s'accumulent peu à peu des commentaires, dont certains résistent à l'usure du temps jusqu'à fournir ces raisons fortes qu'invoque Boudon face aux tenants de la déconstruction ou de l'interprétation sans fin. Aussi une sociologie des arts centrée sur la façon dont sont reçus les œuvres ou les artistes, sur les constantes comme sur les variations, sur les accords 
comme sur les désaccords, sur les faits comme sur les discours, s'avère un projet scientifique non seulement défendable, mais encore fructueux ainsi que le montrent divers travaux récents qui, s'ils s'inscrivent dans une perspective constructiviste $^{4}$ ou relationniste ${ }^{5}$, ne relèvent ni d'une visée nihiliste ni d'une épistémologie relativiste.

\section{DE LA GLOIRE}

Ainsi Nathalie Heinich aborde-t-elle les textes, les propos, l'iconographie, ou encore les comportements qu'engendrent la vie et l'œuvre de Van Gogh depuis un siècle comme autant de matériaux à partir desquels tenter un essai d'anthropologie de l'admiration. Plutôt que d'expliquer l'aura qui entoure le peintre par les qualités intrinsèques de sa peinture ou, à l'inverse, de se gausser des louanges dont l'un et l'autre font l'objet, elle se propose d'étudier la façon dont s'exerce une admiration qui confine à la vénération. D'emblée, elle s'attache à montrer que, de son vivant déjà, et contrairement à une idée reçue, la peinture du hollandais éveille l'intérêt de la critique. Une analyse détaillée des articles parus jusqu'en 1901 permet d'apercevoir «comment un ensemble d'images a été constitué en œuvre, et cette œuvre en une série de chefs-d'œuvre» (Heinich, 1991: 18). En un temps où les critères de l'excellence artistique que fournit la tradition académique, s'ils sont menacés, n'ont pas encore été véritablement remplacés, les termes dans lesquels la critique fait l'éloge ou - ce qui est plus rare - dénigre cette peinture s'avèrent significatifs: Van Gogh serait unique en son genre, et sa vie, l'illustration des tourments qui assaillent le créateur authentique.

Mais pour faire d'un ensemble de travaux une œuvre, il ne suffit pas d'en souligner l'originalité, encore faut-il l'inscrire dans l'histoire de l'art, ce à quoi s'emploie encore la critique en situant cette démarche par rapport, d'une part, à la peinture du Siècle d'or hollandais et, d'autre part, à l'impressionnisme ou au symbolisme. Ce «double mouvement contraire de particularisation et de généralisation» (Heinich, 1991: 42) marque l'instauration d'un espace herméneutique dont l'enjeu est le sens conféré à l'œuvre de Van Gogh. Il apparaît alors que toute interprétation requiert certaines conditions de possibilité, à commencer par la présence d'une énigme susceptible de retenir l'attention. Mais il faut encore que l'œuvre présente une relative cohérence, qu'elle procède de l'inspiration plutôt que de l'imitation et qu'elle recèle une valeur universelle. Reconnaître ces qualités à la peinture de Van Gogh revient à participer, fût-ce malgré soi, au processus de «construction d'une singularité digne d'admiration» (Heinich, 1991: 47) - processus pour lequel une décennie après la disparition du peintre aura suffi.

\footnotetext{
Ainsi que le rappelle Philippe Corcuff (199: 19), «dire qu'une maison est 'construite' signifie simplement qu'elle est le résultat d'un travail humain et qu'elle n'a pas été là de toute éternité, et non qu'elle n'existe pas, bien au contraire.» Et d'ajouter: «Les constructivismes sont de nouvelles formes de réalisme, se distinguant toutefois des formes classiques de positivisme, car interrogeant le 'donné' et laissant place à une pluralité de réalités dont les relations doivent être pensées ».

s Selon Frédéric Vandenberghe (2001 : 41), la sociologie devrait à Karl Mannheim l'introduction de ce néologisme dans son vocabulaire.
} 
La vérité des faits une fois établie, il reste à expliquer pourquoi, selon cette légende, il faut que Van Gogh ait été un incompris. Si le nombre des publications consacrées à sa vie ne cesse de croître depuis le début du $\mathrm{XX}^{\mathrm{e}}$ siècle sans apporter beaucoup d'informations inédites, certains motifs biographiques reviennent avec insistance: la vocation, le génie, la solitude, la pauvreté, l'incompréhension, le martyre. Or plusieurs de ces motifs caractérisent les «vies de saints » tels que les rapporte la tradition catholique et, pour certains d'entre eux, ils se fondent sur ce que fut effectivement l'existence de Van Gogh ainsi que le montre la correspondance avec son frère Théo, emplie d'allusions à l'imbrication entre expérience religieuse et création artistique, des vertus de l'ascèse à l'amour du prochain. L'artiste se tranche l'oreille et, plus tard, il se suicide, ce qui en fait un martyr de l'art aux yeux de biographes dont beaucoup cèdent à l'hagiographie et l'érigent en modèle de sainteté, auréolé de cette gloire dont la peinture du XVIII ${ }^{\mathrm{e}}$ siècle avait pour habitude d'envelopper le corps du Christ. On assisterait là à un «déplacement vers le domaine artistique des formes religieuses de l'excellence» (Heinich, 1991 : 92), déplacement dont la vie de l'artiste contiendrait les prémisses sans en fournir toutes les raisons.

Stigmatiser ces excès hagiographiques revient en règle générale à insister sur l'œuvre plutôt que sur la personne de l'artiste. Prendre ainsi ses distances, adopter une posture critique, ce n'est toutefois qu'occuper une position parmi d'autres: celle où la célébration - à laquelle aucun discours n'échappe - prend le visage de la réhabilitation. Il apparaît alors que ce qui sépare le propos des spécialistes de celui que perpétue la légende ne se résume pas à leur exactitude respective. Par contre, l'antinomie est claire entre «le discours savant, qui privilégie l'herméneutique de l'œuvre géniale, et le sens commun, qui privilégie l'hagiographie de la personne sanctifiée» (Heinich, 1991: 102), - opposition qu'on retrouve dans le cas d'un écrivain comme Kafka, dont les commentateurs avertis analyseront l'écriture sans la rapporter si ce n'est incidemment à la vie de l'auteur. Quant à l'épreuve de la folie qu'aura affrontée Van Gogh, ses biographes, ses critiques, divers psychiatres même ne s'emparent de ce motif qu'à dater des années vingt. La multiplicité des diagnostics alors posés prête à sourire, mais si l'analyse s'annonce sans fin, c'est que «la folie du peintre est d'autant plus intéressante que sa peinture est plus valorisée» (Heinich, 1991: 122). La période est aussi celle où apparaît le surréalisme, celle où s'affirme la psychanalyse, celle où il est encore possible de discuter du lien entre production artistique et aliénation mentale sans que la toile coure le risque de se voir reléguée au rayon de l'art dégénéré. Tour à tour admiré en tant que génie, saint ou héros, la grandeur de Van Gogh serait d'avoir tout donné, et jusqu'à sa raison, pour la peinture si bien que «plus il est dit fou par les psychiatres, plus il peut être dit grand par les admirateurs » (Heinich, 1991: 133). Même Antonin Artaud, s'il dénie la folie du peintre, le dira grand parce que «suicidé de la société », victime d'un aveuglement collectif. Dès lors, la faute de sa mort à l'âge de trente-sept ans rejaillirait sur la société tout entière, condamnée à rembourser sa dette et à expier l'inexpiable.

Bientôt se multiplieront ainsi les hommages, les biographies, les chansons, ou encore les films consacrés à l'œuvre et à la personne de Van Gogh. L'envol du prix de ses toiles en salles des ventes - dont s'indignent ses admirateurs parce que sans commune mesure avec les difficultés que connut le peintre - devrait être compris comme «la forme marchande d'un rachat de l'injustice commise envers la 
personne» (Heinich, 1991: 164). Il en va de même de la visite aux œuvres, qui relèverait carrément de l'ordre du «miracle»: durant l'année du centenaire, en 1990, des foules énormes accoururent aux Pays-Bas. Plus nombreux étaient les gens à s'agglutiner devant les œuvres de ce grand peintre, plus grand était l'auteur de ces toiles devant lesquelles on se pressait. Là encore, il ne suffit pas de dénoncer ce comportement vulgaire, cette folie ou encore la récupération opérée par l'institution muséale. Si dénonciation il y eut, comme en témoignent nombre d'articles de presse qui en appelèrent à une relation plus authentique à l'œuvre et à la personne, «c'est que l'admiration, justement, n'est pas la reconnaissance: c'est l'amour ajouté à la reconnaissance » (Heinich, 1991: 177). Aux yeux des admirateurs du peintre, l'admiration, comme la foi, ne connaît pas de limites: on n'admire ni ne croit jamais assez.

Poussée jusqu'à son terme, la thèse que défend Nathalie Heinich l'entraîne en conclusion à inscrire la manifestation organisée le 28 juillet 1990 à Auvers-surOise, où est enterré le peintre, au registre des processions au corps. Elle se fait alors ethnographe et décrit avec moult détails ce que fut cette commémoration: une cérémonie avec ses discours, ses cortèges, sa marche silencieuse, mais aussi sa contre-manifestation qui tournait en dérision le pèlerinage officiel. Ce jour-là, la communauté des fidèles, unis dans la célébration du disparu, se serait efforcée de racheter par sa présence la dette contractée à l'égard de Vincent...

\section{DU GÉNIE}

Une telle démonstration, à la fois systématique et incisive, ne convainc cependant pas totalement dès l'instant où, de manière délibérée, elle ne fait aucun cas, sinon de manière indirecte, des œuvres produites par Van Gogh. Seule compte la façon dont ses tableaux furent reçus, leur étude plus précise demeurant réservée à ces spécialistes de l'œuvre que sont les historiens de l'art. Or ce parti pris méthodologique $^{6}$ permet difficilement d'apercevoir comment telle toile et ses propriétés spécifiques suscitèrent alors - ou suscitent encore - telle ou telle réaction, de la part de qui et dans quelles circonstances. Du même coup, tout semble se passer comme si, parmi les raisons fortes que nous aurions aujourd'hui encore d'admirer Van Gogh, celles issues de l'histoire de sa réception prévalaient sur celles attribuables à la rencontre directe avec son oeuvre, et ce précisément parce qu'il n'est pas de regard sur la peinture qui ne soit tributaire de multiples filtres s'interposant entre sujet et objet.

Ceci dit, la voie qu'emprunte Nathalie Heinich débouche sur des résultats non négligeables, qu'il s'agisse du rôle joué par la critique d'art, des catégories que celle-ci met en œuvre, ou encore de ce genre littéraire singulier que constituent les biographies d'artiste. La mise en relation d'arguments contradictoires de sorte, d'une part, à épuiser l'espace des interprétations auxquelles l'œuvre et la personne de Van Gogh donnèrent lieu et, de l'autre, à le reconstruire en vue de dégager les thèmes récurrents susceptibles d'apparaître à propos d'autres cas, la conduit à étaler tous ses matériaux sur un même plan. Ainsi son analyse de la réception se

Parti pris méthodologique sur lequel l'auteur s'explique ailleurs (Heinich, 1998) en détail. 
veut-elle d'une parfaite neutralité axiologique en ne prenant parti pour aucune des interprétations que suggère cette peinture. En effet, que nous rejoignions les rangs des dévots ou qu'à l'inverse, nous en dénoncions les excès, nous demeurerions prisonniers d'un cercle herméneutique qu'il appartiendrait à la sociologie, non de briser, mais de retracer.

Considérer les divers points de vue en présence avec la même distance ou la même indifférence revient à assumer une posture qu'il s'avère toutefois équivoque de qualifier - en ce sens circonscrit - de relativiste, aucune volonté de soustraire ses résultats à la critique d'autrui ne pouvant être imputée à un travail qui confirme à l'inverse que, plus cohérente est la démonstration, mieux elle se prête à la discussion.

La remarque qui précède vaut également pour l'ouvrage que Tia DeNora a plus récemment consacré à Ludwig van Beethoven (DeNora, 1998) et où elle s'efforce de comprendre le succès que rencontre sa musique dès son arrivée à Vienne à la fin du XVIII ${ }^{\mathrm{e}}$ siècle. Prenant à contre-pied la légende du génie méconnu telle que la forgera le $\mathrm{XIX}^{\mathrm{e}}$ siècle, Tia DeNora retrace avec minutie les conditions dans lesquelles le jeune pianiste travaille, d'abord auprès de Haydn, puis en s'appuyant sur le soutien que lui apporte l'aristocratie locale, les relations qu'il tisse avec d'autres musiciens, éditeurs de musique ou organisateurs de concerts, la façon dont il prend position par rapport à la tradition musicale, à Bach ou encore à Mozart. Mais il ne s'agit pas là de réduire la gloire du créateur au monde de l'art dont il dépend, car si tout succès comporte une part d'arbitraire, encore fallait-il être Beethoven pour l'obtenir. Par contre, en montrant comment ce dernier sut placer son talent au service de sa renommée au moment où, en partie sous son influence, de nouveaux canons esthétiques étaient en train d'émerger, Tia DeNora éclaire à la fois les déterminations qui conditionnent toute trajectoire biographique fut-elle celle d'un génie et la capacité qu'a l'artiste de peser sur les termes dans lesquels sera reçue son œuvre.

L'idée selon laquelle il existerait des «classiques» de la musique naît d'ailleurs dans le contexte viennois et les critères au nom desquels reconnaître ces grands maîtres font l'objet d'un débat public dans lequel s'inscrivent à la fois Beethoven, sa musique sérieuse et ses audaces stylistiques. Héritier en rupture de ban, celui-ci quitte Bonn pour Vienne où, précédé d'une réputation flatteuse comme virtuose, il peut compter sur les liens préalablement noués avec une aristocratie locale qui lui apportera protection et liberté. Dès le départ, ce que Pierre Bourdieu aurait nommé son capital social s'avère plutôt consistant, un capital qui ne cessera d'augmenter ainsi qu'en atteste la liste des personnalités auxquelles iront ses dédicaces. Sans le soutien de ces mécènes privés et autres amateurs de musique savante, qui le reçoivent dans leurs salons, souscrivent des abonnements pour ses concerts publics ou avancent les montants nécessaires à l'édition de ses partitions, il eut été vain d'espérer un quelconque succès commercial.

En examinant de près les relations entre Haydn et Beethoven, Tia de Nora montre comment, dans ce contexte, et du vivant même de l'artiste, se construit sa légende, - exercice périlleux s'il en est puisqu'il l'oblige à mettre en doute les biographies autorisées, voire à refuser les conclusions auxquelles parviennent d'éminents musicologues à partir des mêmes sources qu'elle. Ainsi le maître, Haydn, ouvre-t-il des portes à son élève, Beethoven, qui le fait en retour bénéficier de son succès si bien qu'au bout du compte, tous deux voient leurs noms associés 
à l'idée de grande musique par opposition à la musique de divertissement, celle que composent les valets au service et pour le plaisir de leur maître.

Les duels pianistiques qui opposent Beethoven à ses rivaux, les controverses qu'il engendre, les motifs critiques qui s'attachent à sa musique, jugée plus dense, plus complexe que d'autres, font évoluer le goût musical de ses contemporains. Partisans et adversaires échangent leurs arguments tandis qu'une comparaison devient possible entre divers styles, musiciens ou mécènes. Le pianiste - et compositeur - cherche à contrôler les moments et les lieux où il se produit, il impose le silence lorsqu'il joue, il agit en sorte que son originalité soit reconnue. Des contrats qu'il passe avec les éditeurs de musique en les incitant à publier autre chose que des variations à usage domestique aux requêtes qu'il adresse à ses fournisseurs afin d'obtenir un instrument plus sonore et plus robuste, Beethoven se comporte en «entrepreneur social» (DeNora, 1998: 209) soucieux d'élargir son public à une bourgeoisie prospère et en pleine ascension. Aussi l'étude du génie en tant que construction sociale implique-t-elle de tenir compte simultanément de «l'interaction, au fil du temps, des activités artistiques de Beethoven en personne, de l'idée qu'il se faisait de lui-même, et de la construction d'un environnement qui lui soit favorable» (DeNora, 1998: 265).

Focalisée sur l'enchevêtrement de ces relations en vue d'en reconstruire la trame ou d'en comprendre la dynamique, l'analyse sociologique ne signifie en rien diminuer la valeur de l'œuvre ou la grandeur de l'artiste; au contraire, elle permet d'en prendre la mesure, littéralement, de les apprécier. A cet égard, on retiendra que les termes dans lesquels fut reçue la musique de Beethoven auront été très tôt fixés si bien qu'entre les raisons fortes que nous pourrions invoquer pour justifier notre admiration et celles formulées à l'époque, l'écart risque de se révéler mince. Mais ce constat invalide-t-il pour autant l'approche relationnelle adoptée par Tia DeNora? Tout au plus doit-il nous inciter à ne pas faire la part trop belle à l'arbitraire dans le destin de ces oeuvres que nous persistons à considérer comme des sommets de la création. Par ailleurs, le fait d'aborder l'œuvre d'art, non comme un donné sur lequel viendraient se greffer après coup de multiples significations ${ }^{7}$, mais bien comme le produit d'une activité collective d'où, du vivant même de l'artiste et à son initiative, émergent des critères de valeur parmi lesquels certains sont destinés à perdurer, tend à montrer qu'une approche du génie au travers des «mondes de l'art», plutôt que de faire obstacle à un questionnement en termes de «raisons fortes», le rend d'autant plus pertinent ne serait-ce que pour vérifier si l'amour pour Beethoven se décline aujourd'hui encore dans les formes qui furent celles du passé alors même que son écoute passe désormais plus souvent par le compact disc que par les salons de l'aristocratie viennoise. Peut-être découvrirait-on alors qu'entre ces deux approches, l'une «cognitiviste» et l'autre, «constructiviste», les convergences l'emportent sur les divergences si bien que la sociologie des arts n'aurait pas à revenir à Simmel ou à Francastel pour repartir du bon pied?

Cf. sur ce point Hennion, 1997. 


\section{DE LA GRANDEUR}

Ecrit, cette fois, en collaboration entre un musicologue et un sociologue, le troisième livre qui retiendra notre attention a pour ambition, précisément, de comprendre «ce que signifie la grandeur de Bach pour un amateur aujourd'hui et, pour cela, en mener l'archéologie à travers sa formation dans le cas de la France au XIX ${ }^{\mathrm{e}}$ siècle» (Fauquet J.-M., Hennion A., 2000: 10). Ses auteurs se refusent d'emblée à analyser la réception de Bach comme si son œuvre était «déjà là » de sorte qu'il ne resterait plus qu'à en éclairer les diverses interprétations. En bref, d'après eux, il convient d'expliquer à la fois la production d'un goût pour Bach, celle d'une œuvre correspondant à ce goût et celle des dispositifs au travers desquels la goûter selon des modalités qui diffèrent aujourd'hui d'hier. On peut voir là un reproche indirect adressé à la démarche de Nathalie Heinich, qui traitait de l'admiration pour Van Gogh sans jamais mentionner un quelconque tableau, mais aussi à Tia DeNora pour n'avoir pas pris en compte la suite de la carrière de Beethoven et, surtout, ce que le siècle qui suivit sa disparition fit de sa musique. En outre, l'attention qu'accordent Joël-Marie Fauquet et Antoine Hennion aux œuvres elles-mêmes, aux effets qu'elles engendrent et aux voies par lesquelles elle se transmet semble donner raison à ceux qui, comme Demeulenaere, jugent déplacé de les ignorer. Toutefois, leur projet ne consiste pas à chercher au niveau de l'œuvre les raisons pour lesquelles nous l'admirons, mais bien à reconstituer la genèse, la structure et la dynamique des relations au sein de ce ménage à trois que forment œuvres, goûts et médiations sans jamais privilégier l'un ou l'autre de ces pôles.

La musique de Bach, au XIX ${ }^{\mathrm{e}}$ siècle, ce sont certaines pièces plutôt que d'autres, qui plus est, jouées et écoutées de diverses manières. Une musique en permanence réinventée par les usages dont elle fait l'objet, usages au travers desquels évolue le goût musical, en particulier la définition de la musique dite classique parce que sérieuse, noble et propre à élever l'esprit de qui en joue ou l'écoute. Ainsi, de 1800 à 1885, diverses sont les sauces auxquelles Bach se trouve accommodé jusqu'à ce que les traits d'un profil quasi définitif se fixent pour longtemps. Entre-temps se seront succédé les commentaires, les critiques, les transcriptions et autres arrangements de sa musique qui, au même titre que les programmes de concert, les partitions pour amateurs et les exercices utiles à l'apprentissage du piano ou du violon, auront «fabriqué » celui qui, pourtant, fut très tôt considéré comme le père de la musique. Ainsi, pour les musicologues du XIX siècle, Bach aurait tout inventé si bien qu'il ne resterait plus qu'à apprendre à l'aimer: si nous le connaissons si mal, c'est parce que nous ne l'avons pas encore assez étudié. Le voilà du même coup célébré par un premier cercle d'admirateurs - dont Franz Liszt -, lesquels s'emparent de sa musique, l'arrangent, la transcrivent et la jouent en concert. Le clavecin, l'orgue ou le piano à pédalier sont tour à tour utilisés, - l'histoire de la musique étant, aussi, celle de la technique. Entre l'Allemagne et la France, un réseau d'échanges se met en place au travers duquel se diffuse la connaissance de l'œuvre en «installant dans le même geste le goût de Bach et le Bach que l'on goûtera» (Fauquet \& Hennion, 2000: 54).

Puis vient le temps des biographies, d'habitude fondées sur quelques anecdotes, toujours les mêmes, qui composent le récit d'une vie dont la simplicité ou la banalité sont données pour exemplaires tant elles contrastent avec la grandeur 
de l'œuvre. Le musicien est dès lors devenu une figure littéraire. Deux ou trois choses que l'on sait de lui suffisent à l'ériger en maître, sans rival qui soit à sa hauteur, ce qui lui vaut des épithètes d'autant plus élogieuses que ses biographes peinent à trouver les mots en mesure de caractériser son génie. Ouvrages et articles «font» Bach tout au long du XIX ${ }^{\mathrm{e}}$ siècle, ils en accompagnent l'écoute, ils en développent le goût.

Toutefois, s'il est concevable de reconstituer un répertoire ou de délimiter un corpus bibliographique, comment savoir quelles émotions suscitait alors cette musique et quels effets elle exerçait sur ceux qui l'interprétaient ou l'écoutaient? Parmi les sources disponibles, il n'y a guère que les critiques rédigées à la sortie des concerts qui puissent être exploitées, qu'il s'agisse des réactions à chaud ou des commentaires de la presse musicale. Ce que disent les critiques et comment ils le disent présente donc beaucoup d'intérêt même si, le plus souvent, ces derniers «s'arrêtent peu sur l'œuvre, pour remonter bien vite à leur auteur, d'un côté, ou s'attarder sur les interprètes, de l'autre: les personnes sont plus faciles à louer et à blâmer que la musique à qualifier » (Fauquet \& Hennion, 2000: 141). On observe néanmoins une lente cristallisation - ou décantation? - du discours critique qui entoure Bach et sa musique au fur et à mesure que celle-ci est mieux connue. Les mêmes arguments reviennent par exemple dans les comptes rendus qui suivent l'exécution de ces pièces emblématiques que sont devenus le Clavier bien tempéré ou la Passion selon saint Matthieu. Seules quelques voix discordantes se font entendre pour regretter le caractère par trop dépouillé de cette musique ou certaines de ses longueurs, mais en règle générale, les thèmes qui font aujourd'hui encore la réputation du compositeur sont ceux qui furent reconnus comme majeurs à l'époque.

Parallèlement se développe un public d'amateurs qui prend plaisir à l'écoute de la musique dite classique pour laquelle, selon la loi de l'offre et de la demande, s'organise un marché sur lequel s'activent des musiciens professionnels, libérés de la tutelle du prince ou de l'église, mais aussi des organisateurs de concerts, des éditeurs, des imprimeurs, ou encore des facteurs d'instruments. De voie d'accès vers Dieu lorsqu'elle était jouée durant le culte dominical au temple de Leipzig, la musique de Bach est devenue un objet de délectation, une œuvre à déguster en public et, bientôt, grâce à la radiophonie et à l'industrie du disque, en privé. Ce que désormais nous écoutons et la manière dont nous l'écoutons résultent d'un long travail collectif au terme duquel Bach est «en nous» (Fauquet \& Hennion, 2000: 207), à la fois familier et omniprésent, connu par cœur au point que seule fait encore problème la fidélité des interprètes ou des enregistrements à des origines à jamais inaccessibles, mais jamais l'estime que cette musique mérite par delà les diverses cultures ou religions. L'admiration pour elle, désormais, est universelle.

\section{CONCLUSION}

Qu'il s'agisse de Van Gogh, de Beethoven ou de Bach, aucun des auteurs qui précèdent ne se risque à faire l'impasse sur le rôle que jouent les œuvres dans l'admiration que nous leur portons. Les raisons fortes qu'à leur vue ou à leur écoute, nous donnerions de les apprécier, jamais ils ne les traitent comme des illusions. Le 
fait que ces raisons nous semblent aller de soi au point de s'étonner que d'autres puissent ne pas les partager, ils l'acceptent volontiers. Mais ils se refusent à ne pas chercher d'explication autre à cette situation que celle concluant que, si le chefd'œuvre est dans les esprits, c'est qu'il est d'abord dans la chose de sorte que le fait de s'interroger sur l'émergence, la variabilité ou l'enracinement des valeurs qui nous sont parfois les plus chères conduirait au mieux, à perdre son temps, au pire, à sacrifier au relativisme ambiant.

Le polythéisme des valeurs, si l'on accepte le diagnostic webérien, n'empêche personne de préférer un prélude de Frédéric Chopin à un rock de Chuck Berry, ou le contraire, ou encore de les aimer tous les deux. Quel que soit le cas de figure, nos choix se fonderont sur des raisons solides et largement partagées, raisons dont on conçoit mal au nom de quels principes certaines en excluraient d'autres. En dresser l'inventaire, en reconstituer la genèse, en montrer la prégnance ou la récurrence, bien loin de déboucher sur la conclusion qu'il n'y aurait là qu'arbitraire, croyance ou illusion, permet de comprendre par quelles voies passe notre admiration et, du même coup, celle d'autrui.

Le fait que chaque œuvre d'art possède des qualités propres qu'il serait absurde de passer sous silence, mais qu'en même temps elle se prête à de multiples interprétations, conduit le philosophe Yves Michaud à argumenter en faveur de ce qu'il nomme un «relativisme mesuré et conceptuellement organisé» (Michaud, 1999: 19-20) puisque, écrit-il, «il n’y a aucune raison que le pluralisme dont nous avons à tenir compte pour être fidèle aux faits débouche sur un relativisme du 'tout se vaut'»(Michaud, 1999: 13). Bien plus, s'il remarque combien nos jugements peuvent être divers, il souligne aussi qu'en matière esthétique, les accords sont plus fréquents qu'une telle diversité pourrait le laisser penser. Aussi nous invite-t-il, à l'instar de Raymond Boudon, à nous pencher sur l'émergence et sur la cristallisation de la norme tout en concédant que «si c'est finalement toujours dans une relation directe que nous ressentons et évaluons l'œuvre d'art, cette expérience directe a toujours été précédée de médiations qui aménagent et rendent possible la relation directe en produisant son contexte et ses conditions de signification» (Michaud, 1999: 24).

Voilà pourquoi, s'il n'est nul besoin d'elle pour goûter la peinture de Van Gogh ou la musique de Beethoven, la sociologie des arts concourt à les aimer en meilleure connaissance de cause.

Département de sociologie

Université de Genève

\section{RÉFÉRENCES BIBLIOGRAPHIQUES}

Boudon, R. (1994), «De l'objectivité des valeurs artistiques ou les valeurs artistiques entre le platonisme et le conventionnalisme», in: Le sens des valeurs, Paris, PUF.

Boudon, R. (1995), «La nouvelle sociologie de la connaissance scientifique», in: Le juste et le vrai. Études sur l'objectivité des valeurs et de la connaissance, Paris, Fayard.

Boudon, R. (1996), «Relativisme et modernité», Revue européenne des sciences sociales, XXXIV, $106,169-192$. 
Bouvier, A. (1998), «Argumentation et évaluation esthétiques du point de vue d'une sociologie cognitive», in: S. Mesure (Ed.), La rationalité des valeurs, Paris, PUF.

Coenen-Huther, J. (1999), «L'homo sociologicus et ses variétés», Revue européenne des sciences sociales, XXXVII, 113, 87-102.

Coenen-Huther, J. (2001), «Sens moral ou raisons fortes?», L'Année sociologique, 51, 1, 233-254.

Corcuff, P. (1995), Les nouvelles sociologies, Paris, Nathan.

Demeulenaere, P. (1998), «La différence entre le relativisme des valeurs et la pluralité des valeurs du point de vue d'une sociologie de l'art », in: S. Mesure (Ed.), La rationalité des valeurs, Paris, PUF.

DeNora, T. (1998), Beethoven et la construction du génie. Musique et société à Vienne 1792-1803, Paris, Fayard.

Fauquet, J.-M., Hennion, A. (2000), La grandeur de Bach. L'amour de la musique en France au XIX siècle, Paris, Fayard.

Heinich, N. (1991), La gloire de Van Gogh. Essai d'anthropologie de l'admiration, Paris, Minuit.

Heinich, N. (1998), Ce que l'art fait à la sociologie, Paris, Minuit.

Hennion, A. (1997), «La sociologie est-elle définitivement allergique au génie? Pour un programme constructiviste fort en sociologie de l'art», in: B. Dorléac, L. Gervereau, S. Guilbaut \& G. Monnier (Eds), Où va l' histoire de l'art contemporain?, Paris, ENSBA, 230-240.

Lang, G. E., Lang, K. (1990), Etched in Memory. The Building and Survival of Artistic Reputation, Chapel Hill/London, The University of North Carolina Press.

Marx, K., Engels, F. (1954), Sur la littérature et l'art. Textes choisis, Paris, Editions sociales.

Michaud, Y. (1999), Critères esthétiques et jugements de goût, Nîmes, Jacqueline Chambon.

Steiner, G. (1998), Errata. Récits d' une pensée, Paris, Gallimard.

Vandenberghe, F. (2001), «Relativisme, relationnisme, structuralisme», Simmel Studies, 12, 1, 2002, 41-84. 